\title{
Growth Through Loss After Brain Injury
}

\author{
Tamara Ownsworth ${ }^{1}$ and Jennifer Fleming $2,3,4$ \\ ${ }^{1}$ Griffith University, Australia \\ ${ }^{2}$ The University of Queensland, Australia \\ ${ }^{3}$ Occupational Therapy Department, Princess Alexandra Hospital, Australia \\ ${ }^{4}$ Centre for Functioning and Health Research, Metro South Health Services District, Australia
}

$I^{1}$ is well recognised that people can derive meaning from and experience positive psychological changes even in the most adverse circumstances surrounding illness and trauma. Although many different theories of 'posttraumatic growth' (PTG) exist in the literature (see Zoellner \& Maercker, 2006), there is consensus that when people experience an event as traumatic or highly stressful, their global assumptions about themselves and the world can be shattered. Those who perceive the impact of the event to be more threatening (or less congruent with their prior beliefs and values) are more likely to experience psychological distress. Such distress can propel a series of introspective processes and coping behaviours in efforts to make sense of the loss. In time, these processes can restore one's sense of meaning in life and lead to fundamental changes in values and directions in life, self-identity, relationships and a deepened spirituality (Park, 2010; Tedeschi \& Calhoun, 2004; Zoellner \& Maercker, 2006).

Psychological growth is not inevitable after trauma, however, and distress can often be endured without the person understanding its meaning or drawing psychological benefits. Tedeschi and Calhoun's (2004) theory of PTG argues that effortful cognitive processes (e.g., reanalysis and reinterpretation of the event and its meaning) play a key role in transforming people's beliefs about themselves and the world. Hence, the extent to which this can occur and factors influencing PTG for people with significant neurological compromise after brain injury is intriguing from a theoretical viewpoint. Further, given that greater PTG is associated with better mental health in the long term (Helgeson, Reynolds, \& Tomich, 2006) a more advanced understanding of meaning-making processes after acquired brain injury is essential to inform psychological interventions.
Brain injury is without doubt one of the most life-altering events a person can experience. The immediate circumstances of the injury are often life-threatening, while the ongoing physical, cognitive and behavioural consequences can threaten independence, relationships, livelihood and sense of self. It is perhaps because of the well-recognised adverse consequences, including depression and psychosocial dysfunction, that positive psychological changes after brain injury have received little attention in the literature. Although some authors have investigated successful adaptation and positive outcomes after brain injury (e.g., Gillen, 2005; Nochi, 2000; Strang \& Strang, 2001), such studies are rare for brain injury compared to other chronic and life-altering health conditions. There is a small body of relatively recent studies that support the view that people can experience positive changes in their outlook and behaviour, an enhanced sense of self and stronger relationships in spite of, or because of their brain injury (Collicutt McGrath \& Linley, 2006; Collicutt McGrath, 2008; Gangstad, Norman, \& Barton, 2009; Nochi, 2000; Powell et al., 2007; Strang \& Strang, 2001). The focus on PTG as part of a positive psychology perspective represents a promising area of research and clinical practice in brain injury rehabilitation.

This special issue is devoted to the concept of PTG after brain injury, with articles spanning the theoretical foundations of PTG and positive psychology, empirical investigations of the nature of and circumstances promoting PTG, and psychological, social and occupational interventions for enhancing growth and engagement in meaningful activities and life roles. 


\section{Conceptual Issues and Empirical Studies on PTG}

The initial section of the issue focuses on conceptual foundations and empirical investigations of PTG following ABI. The introductory article is by Revd Dr Collicutt McGrath who was the first researcher to systematically assess PTG after brain injury (Collicutt McGrath \& Linley, 2006). In her review on PTG and spirituality she advocates that PTG is distinct from recovery and resilience and refers to self-reported personal development that extends beyond one's pretrauma level of functioning. Her theoretical account argues that PTG can be viewed as either the outcome of successful psychological accommodation to trauma or a means of coping (e.g., positive illusions and benefit finding). Collicutt McGrath highlights the interrelatedness of PTG and spirituality in which both involve engaging with the existential significance and the transcendence of crises and trauma. Suggestions are made for professionals to identify and support individuals' psychospiritual needs in rehabilitation.

The first empirical article by Silva, Ownsworth, Shields and Fleming examines factors related to PTG at 6-months postdischarge following brain injury $(N=60)$. Greater subjective impairment at discharge was found to predict higher overall PTG at follow-up. Growth was most evident in the domain of appreciation for life and was positively associated with subjective impairment and depression. Such findings suggest that the perceived functional impact of brain injury plays a key role in stimulating broader meaning making processes after ABI. However, higher levels of both subjective impairment and distress appear to promote the reevaluation of one's priorities and values in life during the early stage of community reintegration after brain injury.

Along a similar vein, Kangas and colleagues investigate factors related to benefit finding in adults treated for benign meningioma. In the overall sample $(N=70)$, greater benefit finding was associated with elevated tumour-related intrusions and avoidance as well as depression. However, time since diagnosis appeared to influence the associations among these variables. Specifically, benefit finding was significantly related to higher depressive symptoms only for people diagnosed within a 2-year period. Additionally, benefit finding was significantly associated with tumourrelated avoidance only for those diagnosed more than 2 years ago. Consistent with theories of PTG, these findings suggest that individuals' efforts to find meaning in their illness are associated with more distress initially. However, in the more longterm adjustment phase, the use of avoidance may reflect a biased self-enhancement coping strategy that supports individuals to derive benefits from their illness.

\section{Interventions to Promote Growth and Engagement}

To introduce the intervention section of this issue, Evans presents an in-depth review of positive psychology (PP), as well as its overlap with and potential applications in brain injury rehabilitation. The key focus of PP is to understand positive factors conducive to happiness and wellbeing and to promote intervention strategies for optimising pleasure, engagement and meaning. Hence, in the context of brain injury a PP perspective shifts the focus from impairment and emotional dysfunction, or fixing what's wrong, to building what's strong. Evans argues that the core values of PP and brain injury rehabilitation are similar, but also notes that an individual's capacity to live a life that is pleasurable, engaging and meaningful can be greatly compromised by the consequences of brain injury. Nonetheless, Evans concludes that PP may offer a useful framework for understanding both positive and negative mental health changes after brain injury, as well as some novel intervention strategies for improving wellbeing.

Ashworth, Gracey and Gilbert's article on Compassion Focused Therapy (CFT) provides an illustration of the applications of PP to brain injury rehabilitation. As outlined in their article, CFT is based on a model of affect regulation that evolved from social, evolutionary and neurophysiology approaches. A key premise of CFT is that emotion regulation difficulties (e.g., self-criticism and worry) activate and are maintained by the threat system and can be reduced by stimulating the soothing-contentment system to produce positive emotions (e.g., selfcompassion and calmness). The authors present a case conceptualisation and description of how CFT techniques were incorporated into a holistic neuropsychological rehabilitation program for 'Jenny' a woman with brain injury. Their preliminary data support the efficacy of CFT for improving mental health and self-esteem. The authors conclude more generally that CFT may represent a promising alternative or adjunct to other cognitive-behavioural therapy approaches.

In another article focusing on interventions to facilitate growth after brain injury, Carbonneau, Martineau, Andre and Dawson describe an occupation-based approach that specifically targets meaningful leisure participation. They conducted a 10-week Leisure Education Program with three individuals with traumatic brain injury to determine if it enhanced participation in and satisfaction with 
leisure, as well as leisure self-efficacy. Using a mixed methods design with pre-, post- and followup assessments, the authors illustrate the positive effects of this program with both standardised questionnaire and qualitative data. For at least two of the participants, these gains extended beyond the domain of leisure to more general improvements in wellbeing, community integration and quality of life. Notwithstanding the fact that this was a small pilot study, the findings suggest that a leisure focus in rehabilitation may be a pathway to promoting growth following traumatic brain injury.

In the final article of this issue, Kennedy, Turner and Kendall use qualitative case study methodology to describe the experiences of two peer leaders and one lay leader (family member) of the Skills to Enable People and Communities (STEPS) program. STEPS is a well-established program in Queensland, Australia that aims to create sustainable networks of support for people with brain injury and their families. This study employs cross-case pattern analysis to explore the process of becoming program leaders and outcomes of this role. In all three cases, the desire to support others was central to the decision to become a STEPS Program Leader. In turn, the program leaders derived many personal benefits or growth outcomes, including an expansion of social roles and skills, improved interpersonal relationships and communication and 'growth in self as a person'. Overall, their findings highlight that through providing opportunities for leadership, personal development and community engagement, social system interventions such as the STEPS program can foster a 'growth viewpoint' for individuals with brain injury and their family members.

The series of articles in this special issue collectively underscore the importance of further research into the nature of PTG and its relevance to clinical practice in brain injury rehabilitation. Research focusing on the role of personal characteristics (e.g., cognitive impairment, coping, personality) and social factors (e.g., social support, group membership, rehabilitation) may assist in identifying the conditions most conducive for PTG.
More significantly, being guided by PTG frameworks and other positive psychology perspectives in rehabilitation has the potential to balance the focus between treating impairments and dysfunction, and identifying and promoting personal strength and meaning after brain injury.

\section{References}

Collicutt McGrath, J., \& Linley, P. A. (2006). Post-traumatic growth in acquired brain injury: A preliminary small scale study. Brain Injury, 7, 767-773.

Collicutt McGrath, J. (2008). Recovery from brain injury and positive rehabilitation practice. In $\mathrm{S}$. Joseph \& P.A. Linley (Eds). Trauma, recovery and growth: Positive psychological perspectives (pp. 259-274). New Jersey: John Wiley \& Sons.

Gangstad, B., Norman, P., \& Barton, J. (2009). Cognitive processing and posttraumatic growth after stroke. Rehabilitation Psychology, 54, 69-75.

Gillen, G. (2005). Positive consequences of surviving stroke. American Journal of Occupational Therapy, 59, 346-350.

Helgeson, V.S., Reynolds, K.A., \& Tomich, P.L. (2006). A meta-analytic review of benefit finding and growth. Journal of Consulting and Clinical Psychology, 75, 797-816.

Nochi, M. (2000). Reconstructing self-narratives in coping with traumatic brain injury. Social Science \& Medicine, 51, 1795-1804.

Park, C.L. (2010). Making sense of the meaning literature: An integrative review of meaning making and its effects on adjustment to stressful life events. Psychological Bulletin, 136, 257-301.

Powell, T., Ekin-Wood, A., \& Collin, C. (2007). Posttraumatic growth after head injury: A long-term follow-up. Brain Injury, 21, 31-38.

Strang, S., \& Strang, P. (2001). Spiritual thoughts, coping and 'sense of coherence' in brain tumour patients and their spouses. Palliative Medicine, 15, 127-34.

Tedeschi, G. \& Calhoun, L.G. (2004). Posttraumatic growth: Conceptual foundations and empirical evidence. Psychological Inquiry, 15, 1-18.

Zoellner, T., \& Maercker, A. (2006). Posttraumatic growth in clinical psychology: A critical review and introduction of a two component model. Clinical Psychology Review, 26, 626-653. 